\title{
Characteristics and mechanism of reciprocal ST-segment depression in acute ST segment elevation myocardial infarction
}

\author{
Qijun Gao MD, Fangfang Bie MD, Yinfu Hu MD, Yafeng Chen MD, Bo Yang MD. \\ Department of Cardiology,First People's Hospital of Jingmen,Jingmen, Hubei Province,P.R. China (Qijun Gao, \\ Fangfang Bie, Yinfu Hu, Yafeng Chen)
}

Department of Cardiology, Renmin Hospital of Wuhan University, Wuhan, Hubei Province, P.R. China (Bo Yang )

Corresponding author:

Name: Yafeng Chen

E-mail: chenyafeng2020@ sina.com phone number 086-917108168

Address: Department of Cardiology,First People's Hospital of Jingmen, Xiangshan Road 168, Jingmen, Hubei

Province,P.R. China 448000

word count : 2050 


\section{Abstract:}

Background:There are few studies on the characteristics and mechanism of reciprocal ST-segment depression(RSTD) in acute myocardial infarction.

Methods:

The electrocardiogram and angiography of 85 STEMI patients were retrospectively analyzed to summarize the characteristics of ST segment changes and explore the mechanism of RSTD.

Results:

A total of 85 patients were included, of which 75 were patients with RSTD (10 patients with anterior myocardial infarction had no RSTD), all 45 patients with inferior myocardial infarction had limb leads RSTD, and 37 of them had anterior lead ST segment depression. Thirty patients with anterior myocardial infarction were accompanied by mild ST segment changes in the limb leads. According to the characteristics of RSTD, it is speculated that the mechanism of RSTD is that the action potential of infarct area decreased, which could not offset the action potential in non-infarct area. All ST segment changes in STEMI can be explained by the proposed mechanism, and the value of the ST segment changes can be calculated in limb leads.

Conclusion: the mechanism of RSTD in acute myocardial infarction is that the negative electrode action potential of the lead was weakened or disappeared, and the positive electrode action potential could not be completely offset, resulting in ST segment depression.

Key words: Mechanism, reciprocal ST-segment depression,electrocardiogram, AMI. 
medRxiv preprint doi: https://doi.org/10.1101/2021.09.21.21263421; this version posted October 7, 2021. The copyright holder for this preprint (which was not certified by peer review) is the author/funder, who has granted medRxiv a license to display the preprint in perpetuity.

It is made available under a CC-BY-NC-ND 4.0 International license .

\section{Introduction}

Acute ST segment elevation myocardial infarction(STEMI) is often accompanied by ST segment depression in the leads opposite to the leads showing ST segment elevation, known as reciprocal ST segment depression (RSTD), which is seen in either ischemia at distance or mirror reflection of ST segment elevation.[1] The RSTD mentioned in this paper refers to mirror reflection of ST segment elevation. The mechanism of RSTD is not clear at present.[2]

In this study, the ECGs of patients with acute STEMI in our hospital were analyzed to summarize the characteristics of RSTD and explore the mechanism of RSTD.

\section{Patients and Methods:}

Study population:

The study design is a retrospective analysis of patients admitted to a tertiary hospital (No.1 Hospital of Jingmen City, Hubei Province, China) from January 1, 2018 to March 31, 2021. The study population included patients presenting to the emergency department with symptoms of acute coronary syndrome (ACS) and ST segment elevation in their ECGs on admission.The study was approved by the No.1 Hospital of Jingmen ethics committee and oral consent was obtained from patients.

\section{Method:}

All patients subsequently underwent emergency coronary angiographies. The ECG and cardiac catheterization images were retrospectively analyzed by cardiologists.

The diagnosis of STEMI was based on typical chest pains or chest tightness with ST segment elevation greater than $0.1 \mathrm{mV}$ (greater than $0.2 \mathrm{mV}$ for $\mathrm{V} 1-3$ leads) in two consecutive adjacent leads and an elevated hsTNI after admission. RSTD was defined as ST segment depression > $0.03 \mathrm{mV}$ in any leads, $40 \mathrm{~ms}$ after the J-point. Patients with non-coronary artery disease or multi-vessel disease were excluded.

Study protocol:

Patients were divided into four groups according to their ECG on admission: 
1. The anterior STEMI without RSTD; 2. The anterior STEMI with RSTD; 3. Inferior STEMI with RSTD in the anterior lead; 4. Inferior STEMI without RSTD in the anterior lead.

The number of each group and the characteristics of the ST segment changes were summarized.

\section{Results:}

A total of 85 patients were included, of which 75 were patients with RSTD (10 patients with anterior myocardial infarction had no RSTD), all 45 patients with inferior myocardial infarction had limb leads RSTD, and 37 of them had anterior lead ST segment depression.

Thirty patients with anterior myocardial infarction were accompanied by mild ST segment changes in the limb leads. (ST segment depression $\leq 0.1 \mathrm{mV}$ appeared in 25 patients and ST segment depression of $0.1-0.2 \mathrm{mV}$ appeared in 5 patients). Twenty-five of the patients combined with inferior ST segment depression. The degree of ST segment depression in lead III was approximately equal to the degree of ST segment elevation in lead aVL. The degree of ST segment depression in lead aVF was about half of that in lead III, and there was no significant depression in lead II(Figure 1). There were 5 cases of anterior myocardial infarction with ST segment elevation in inferior leads (less than $0.1 \mathrm{mV}$ ) and RSTD in aVR or aVL lead.

All ECGs of inferior STEMI had aVL or aVR lead ST segment depression. In 34 cases, the degree of ST segment elevation of lead III was greater than that of lead II(Right coronary artery occlusion was found in 26 cases and circumflex artery occlusion in 8 cases). The degree of ST segment depression of lead aVL was significantly greater than that of lead aVR . In two cases, the degree of ST segment elevation of lead II was greater than that of lead III (circumflex artery occlusion), and in 9 cases, the degree of ST segment elevation of lead II was similar to that of lead III( right coronary artery occlusion was found in 1 case and circumflex artery occlusion in 8 cases). The ST segment depression of lead aVL and lead aVR was also similar, which was about half of the elevations of the ST segment in lead III and lead II. 


\section{Discussion}

To date, the only thing we known about RSTD is mirror reflection of ST segment elevation in acute myocardial infarction, and to our knowledge, this is the first study to explore its mechanism.

Since the electrocardiogram(ECG) records the potential changes of the positive and negative electrodes[3], the reason for the RSTD should be analyzed from the potential change of the positive and negative electrodes of the leads, respectively.

Transmembrane action potential(AP), formed by transmembrane ions, causes periodic changes in the potential inside myocardial cells, as well as reverse periodic changes in the membrane potential $(\sqcup) .{ }^{[4]}$ The membrane potential can be sensed by the electrode. The potential change of positive electrode recorded by the lead is the same as the change in membrane potential $(\mathcal{L})$, while the potential change of negative electrode is the opposite $\left(\curvearrowleft_{-}\right.$, which is similar to the change of intracellular potential. Under normal conditions, all myocardium changes synchronously, there is no current within the myocardium, and this fluctuation of the extracellular potential is not shown on the ECG. When myocardial injury occurs, the action potential (AP) is weakened. The AP of the normal myocardium cannot be offset by the AP of the injured myocardium, and ST segment changes appear. The ST segment of the lead with the injured area as a positive electrode is elevated, while the ST segment of the lead with the injured area as a negative electrode is depressed. Therefore, the mechanism of ST segment elevation and RSTD may be the decrease or disappearance of myocardial action potential in infarct area.

The proposed mechanism explains all ST segment changes in acute myocardial infarction, and the value of the ST segment changes can be calculated in limb leads.

The ST segment changes are determined by the amplitude of the action potential (AAP, the height of the phase 2 plateau of AP), and the reduction of the AAP of the limb electrode can be inferred from the change of the ST segment of the limb leads.

Most of the patients with inferior myocardial infarction were accompanied by right ventricular myocardial infarction. In these patients, the AAP recorded by the leg 
and right arm electrode weakened, and the normal myocardium was recorded by the left arm electrode.

Figure 2 is an ECG of a patient with inferior myocardial infarction and right ventricular myocardial infarction. The ST segment of lead III elevated by $0.7 \mathrm{mV}$ indicates that the leg electrode decreased by $0.7 \mathrm{mV}$ (the positive electrode of lead III is the leg and the negative pole is the left arm). The ST segment of lead II elevated by $0.5 \mathrm{mV}$ indicates that the AAP of the leg electrode was $0.5 \mathrm{mV}$ less than that of the right arm electrode, so the AAP of the right arm electrode was $0.2 \mathrm{mV}$ less than that of the left arm electrode. All the RSTD in limb leads can be calculated by the AAP difference between the positive and negative electrodes. The negative electrode of $\mathrm{aVL}$ is the average value of the right arm and leg electrode, AAP decreased $0.45 \mathrm{mV}$ $[(0.7 \mathrm{mV}+0.2 \mathrm{mV}) / 2]$, and the $\mathrm{ST}$ segment decreased by $0.45 \mathrm{mV}$. The negative electrode of aVR is the average value of the left arm and leg electrode, AAP decreased $0.35 \mathrm{mV}[(0+0.7 \mathrm{mV}) / 2]$, ST segment decreased by $0.15 \mathrm{mV}(0.35 \mathrm{mV}-0.2$ $\mathrm{mV}$ ), and lead I decreased by $0.2 \mathrm{mV}$.

If there was no ST segment change in lead I in inferior infarction, it suggested that the AAP of the left and right arms was equal (the AAP of the left and right arms were not decreased, or decreased to the same degree). Since the negative electrode of $\mathrm{aVL}$ and $\mathrm{aVR}$ is the average value of the leg and contralateral arm, the degree of the ST segment depression is only half of the degree of the ST segment elevation of the inferior lead.

The negative electrode of all leads is the limb electrodes; thus, RSTD should not have appeared in anterior myocardial infarction(Figure 3). However, anterior wall myocardial infarction is often accompanied by lateral or inferior wall myocardial infarction, resulting in decreased AAP of the limb electrode. There were 25 patients with anterior wall myocardial infarction combined with left lateral wall myocardial infarction and 5 patients with inferior wall myocardial infarction. The range of the inferior wall or left lateral wall myocardial infarction in patients with anterior wall 
myocardial infarction is generally small, with little impact on limb electrodes and slight ST segment elevation.

Acute anterolateral wall STEMI leads to a decrease in the action potential of the left arm electrode, which is the negative electrode of lead III, half the negative electrode of the aVF lead, and independent of the lead II. The characteristics of RSTD should be as follows: ST depressions in lead III are the most obvious, there are only half the ST depressions in lead aVF compared with lead III, and no ST depressions appear in lead II. Among the 25 patients with anterolateral STEMI in this study, the findings for 24 patients were fully consistent with our analysis (Figure 1), and the other patient had similar ST segment depression in all inferior leads, suggesting the possibility of myocardial ischemia in the inferior wall or a synchronous decrease in the AAP values recorded by the left and right arm electrodes.

The negative electrode of the precordial leads is the average of the three limb leads. Myocardial infarcts with ST segment elevation of the limb lead are likely to lead to RSTD in the precordial leads. Thirty-seven of the 45 patients with inferior myocardial infarction had precordial leads ST segment depression.

The reason for the absence of RSTD in the precordial leads was that the precordial electrode was close to the infarct area and the AAP was also reduced. Another reason was that the AAP of the inferior myocardium was slightly weakened, which had no obvious effect on the potential of the negative electrode of the precordial lead.

Limitations: It is difficult to obtain patients' local myocardial action potential clinically. So far, there have only been some hypotheses about the mechanism of ST segment elevation, and no mechanism has been proposed for RSTD. Thus, we can only speculate about the mechanism in terms of the characteristics of ST segment change, but the mechanism we have proposed completely conforms to the characteristics of ST segment change, making it the most reasonable and scientific mechanism so far. It is expected that basic research could obtain the local action potential of the myocardium and directly verify the proposed mechanism. 
In summary, the mechanism of RSTD in acute myocardial infarction is that the AP of negative electrode of the lead was weakened or disappeared, and the AP of positive electrode could not be completely offset, resulting in ST segment depression.

\section{Contributorship Statement}

The study was designed by QG and BY, FB collected the ECG,QG drafted the manuscript, YH contributed to critical revision.

\section{Conflict of interest}

The authors declare no potential conflict of interests.

\section{Funding}

This research did not receive any funding.

\section{Reference}

1. Radwan HI, Ahmed AAE, Ammar AS, Roshdy HS. Relation of collateral circulation with reciprocal changes in patients with acute ST-elevation myocardial infarction. J Electrocardiol. 2020 May-Jun;60:36-43. doi: 10.1016/j.jelectrocard.2020.03.011. Epub 2020 Mar 24. PMID: 32247072.

2. Vaidya GN, Antoine S, Imam SH, Kozman H, Smulyan H, Villarreal D. Reciprocal ST-Segment Changes in Myocardial Infarction: Ischemia at Distance Versus Mirror Reflection of ST-Elevation. Am J Med Sci. 2018 Feb;355(2):162-167. doi: 10.1016/j.amjms.2017.09.004. Epub 2017 Sep 18. PMID: 29406044.

3. Bayés de Luna, A. (Ed.) (2012). Ischemia and Necrosis In Clinical electrocardiography (4th ed., pp.

216-270). Chichester, UK: Wiley-Blackwell Publishing.

4. Antoni Bayés de Luna; Diego Goldwasser; Miquel Fiol; Antoni Bayés-Genis, Surface electrocardiography. In: Valentin Fuster, Robert A. Harrington, Jagat Narula, Zubin J. Eapen, Hurst's The Heart, 14e-.McGrawHill, 2017. 
Figure 1. Electrocardiogram of patients with anteriorlateral wall myocardial infarction.

Figure 2. Electrocardiogram of a patient with acute inferior wall ST segment elevation myocardial infarction and right ventricular myocardial infarction.

Figure 3. Electrocardiogram of a patient with acute anterior ST segment elevation myocardial infarction. 
I.

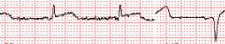

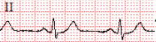

$A^{2}$

III

Avs

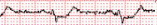

$(1-\cdots)$

$r^{V_{3}} r^{2}-r^{2}-2$

$(-\mathrm{VAR}$

$r^{-3 R}$

f VSR

aVR

4

wrom

$7^{4}$

$+4$

$\sqrt{1} \cdot$

$\longrightarrow u n-\operatorname{mon} u+\cdots$

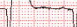

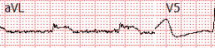

1

vo

v9

ave

var

Undend

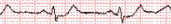

v8

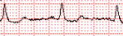




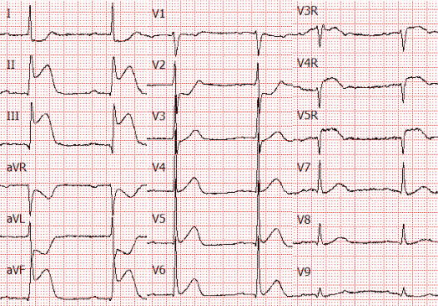




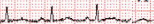

iI $\mathrm{x} 2$

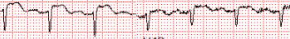

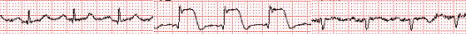
III V3 $\quad$ VSR

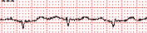

aVR

$\sqrt{4}$

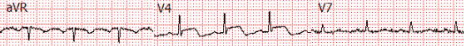

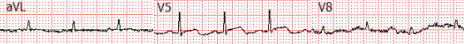
avf v6 ${ }^{a V f}$ 